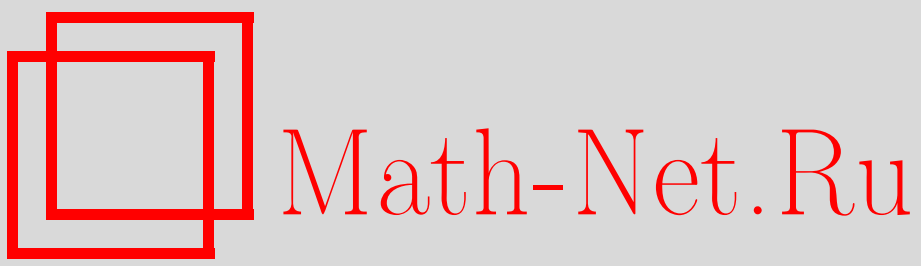

Е. И. Галахов, О достаточных условиях существования полугрупп Феллера, Матем. заметки, 1996, том 60, выпуск 3, 442-444

DOI: https://doi.org/10.4213/mzm1845

Использование Общероссийского математического портала MathNet.Ru подразумевает, что вы прочитали и согласны с пользовательским соглашением

http://www . mathnet.ru/rus/agreement

Параметры загрузки:

IP : 54.197 .130 .99

26 апреля 2023 г., $17: 45: 10$

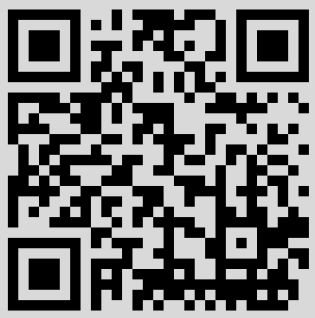




\section{О ДОСТАТОЧНЫХ УСЛОВИЯХ СУЩЕСТВОВАНИЯ ПОЛУГРУПП ФЕЛЛЕРА}

\section{Е.И. Галахов}

Вопрос о существовании неотрицательных сжимающих полугрупп для одномерных диффузионных процессов с нелокальньми краевыми условиями рассматривался в работе В. Феллера [1]. А. Д. Вентцелем [2] было доказано, что если эллиптический дифференциальный оператор второго порядка является инфинитезимальным производящим оператором полугруппы Феллера, то его область определения состоит из функций, удовлетворяющих нелокальньм краевым условиям. Достаточные условия того, чтобы эллиптический оператор с такой областью определения был инфинитезимальным производящим оператором полугруппы Феллера, изучались в работах [3]-[5] и др. При этом авторами предполагалось, что порядок нелокальных членов меньше порядка граничного оператора. Наиболее трудный случай, когда граничные и нелокальные операторы имеют один и тот же порядок, впервые рассматривался А. Л. Скубачевским [6]-[8] при условии убывания нелокальных членов вблизи границы. В настоящей заметке получены достаточные условия существования полугрупп Феллера в предположении, что условие убывания нелокальных членов может нарушаться вблизи некоторого множества точек границы.

1. Пусть $X$ - замкнутое линейное подпространство $C(\bar{Q})$, где $Q \subset \mathbb{R}^{n}$ - ограниченная область с границей $\partial Q \in C^{\infty}, n \geqslant 2$.

ОПРЕДЕЛЕНИЕ 1. Полугруппа $\left\{T_{t}\right\}_{t \geqslant 0}$ линейных ограниченных операторов, действующих на $X$, назьвается полугруппой феллера, если при $t \geqslant 0\left\|T_{t}\right\| \leqslant 1$, a из условия $f(x) \geqslant 0(x \in \bar{Q})$ следует $\left(T_{t}\right) f(x) \geqslant 0(x \in \bar{Q}, t \geqslant 0)$.

Следуя [8], будем рассматривать интегро-дифференциальный оператор $A$ вида

$$
\begin{aligned}
A u= & \sum_{i, j=1}^{n} a_{i j}(x) u_{x_{i} x_{j}}(x)+\sum_{i=1}^{n} a_{i}(x) u_{x_{i}}(x)+a(x) u(x) \\
& -\int_{\bar{Q}}[u(x)-u(y)] m(x, d y) \quad(x \in Q)
\end{aligned}
$$

с областью определения $\mathcal{D}(A)=\left\{u \in C^{2}(Q) \cap C(\bar{Q}): A u \in C(\bar{Q})\right\}$. Здесь $a_{i j}, a_{i}, a \in C^{\infty}\left(\mathbb{R}^{n}\right)$, - вещественнозначные функции, $a_{i j}=a_{j i} ; m(x, \cdot)$ - неотрицательная борелевская мера на $\bar{Q}$.

Обозначим

$$
\begin{gathered}
A^{0} u=\sum_{i, j=1}^{n} a_{i j}(x) u_{x_{i} x_{j}}(x)+\sum_{i=1}^{n} a_{i}(x) u_{x_{i}}(x)+a(x) u(x), \\
A^{1} u=\int_{\bar{Q}} u(y) m(x, d y) .
\end{gathered}
$$

Будем предполагать, что выполнены следующие условия:

1) $\sum_{i, j=1}^{n} a_{i j}(x) \xi_{i} \xi_{j}>0, a(x) \leqslant 0$ для всех $x \in \bar{Q}$ и $0 \neq \xi \in \mathbb{R}^{n}$;

2) линейный оператор $A^{1}$ ограничен в пространстве Гёльдера $C^{\sigma}(\bar{Q})$, где $0<\sigma<1$. 
ЗАмЕчАНИЕ 1. Из условия 2) следует, что $c_{m}=\sup _{x \in \bar{Q}} m(x, \bar{Q})<\infty$. Более того, $m(x, \bar{Q}) \in C^{\sigma}(\bar{Q})$.

Рассмотрим нелокальное граничное условие

$$
B u=\gamma(x) u(x)+\int_{\bar{Q}}[u(x)-u(y)] \mu(x, d y)=0 \quad(x \in \partial Q),
$$

где $\gamma(x) \geqslant 0, \mu(x, \cdot)$ - неотрицательная борелевская мера на $\bar{Q}, \gamma \in C^{\sigma}(\partial Q)$.

Предположим, что выполнены следующие условия:

3) $\gamma(x)+\mu(x, \bar{Q})>0(x \in \partial Q)$;

4) $\partial Q=\Gamma_{1} \cup \Gamma_{2}, \Gamma_{1} \cap \Gamma_{2}=\varnothing$, где $\Gamma_{2}=\{x \in \partial Q: \mu(x, \bar{Q})=0\}$ и внутренность $\Gamma_{2}$ (в топологии границы) непуста;

5) существует такое $\Delta>0$, что для любого $0<\delta<\Delta$ и любого $v \in C^{\sigma}(\bar{Q})$

$$
\int_{\bar{Q} \backslash \Gamma_{1}^{\delta}} v(y) \mu(x, d y) \in C^{\sigma}(\partial Q), \quad \int_{\Gamma_{1}^{\delta}} v(y) \mu(x, d y) \in C^{\sigma}(\partial Q)
$$

и

$$
\begin{gathered}
\left\|\int_{\bar{Q} \backslash \Gamma_{1}^{\delta}} v(y) \mu(x, d y)\right\|_{C^{\sigma}(\partial Q)} \leqslant c_{1}\|v\|_{C^{\sigma}\left(\bar{Q} \backslash \Gamma_{1}^{\delta}\right)}, \\
\left\|\int_{\Gamma_{1}^{\delta}} v(y) \mu(x, d y)\right\|_{C^{\sigma}(\partial Q)} \leqslant c_{2}(\delta)\|v\|_{C^{\sigma}\left(\bar{\Gamma}_{1}^{\delta}\right)},
\end{gathered}
$$

где $\Gamma_{1}^{\delta}=\left\{x \in \mathbb{R}^{n}: \rho\left(x, \Gamma_{1}\right)<\delta\right\}, c_{1}>0$ не зависит от $\delta, c_{2}(\delta) \rightarrow 0$ при $\delta \rightarrow 0,0<\sigma<1$.

ЗАмечание 2 . Пусть $v(y) \equiv 1$. Тогда из условия 5$)$ следует, что

$$
c_{\mu}=\sup _{x \in \partial Q} \mu(x, \bar{Q})<\infty, \quad \mu(x, \bar{Q}) \in C^{\sigma}(\partial Q) .
$$

Следовательно, множество $\Gamma_{2}$ замкнуто, а $\Gamma_{1}$ - открыто в $\partial Q$.

ЗАмечАние 3 . В силу условия 5$) \sup _{x \in \partial Q} \mu\left(x, \Gamma_{1}^{\delta}\right) \leqslant c_{2}(\delta)$. Так как $c_{2}(\delta) \rightarrow 0$ при $\delta \rightarrow 0$, то $\mu\left(x, \Gamma_{1}\right)=0(x \in \partial Q)$.

Обозначим $C_{B}(\bar{Q})=\{u \in C(\bar{Q}): B u=0\}$.

Введем оператор $A_{B}: C_{B}(\bar{Q}) \rightarrow C_{B}(\bar{Q})$ с помощью формул

$$
A_{B} u=A u \quad\left(u \in \mathcal{D}\left(A_{B}\right)\right), \quad \mathcal{D}\left(A_{B}\right)=\left\{u \in C^{2}(Q) \cap C_{B}(\bar{Q}): A u \in C_{B}(\bar{Q})\right\} .
$$

Tеорема 1. Пусть выполнены условия 1)-5).

Тогда оператор $\bar{A}_{B}: C_{B}(\bar{Q}) \rightarrow C_{B}(\bar{Q})$ является инфинитезимальным производящим оператором полугруппы Феллера на $C_{B}(\bar{Q})$, которая однозначно определяется оператором $A_{B}$.

ДОКАЗАТЕЛЬСТВО теоремы основано на использовании теоремы Хилле-Иосиды и дальейшем развитии метода отделения нелокальных членов, использованного в работе [8]. 
ПримеР 1. Рассмотрим следующее граничное условие:

$$
u(x)-\sum_{s=1}^{N} b_{s}(x) u\left(\omega_{s}(x)\right)-\int_{\Omega} c(x, y) u(y) d y=0 \quad(x \in \partial Q) .
$$

Здесь $b_{s} \in C^{\sigma}(\partial Q), c \in C^{\sigma}(\partial Q \times \bar{\Omega}), \omega_{s}$ - непрерьвно дифференцируемые невырожденные отображения некоторой окрестности $V$ границы $\partial Q$ на $\omega_{s}(V)$, такие, что $\omega_{s}(\partial Q) \subset \bar{Q} \backslash \bar{\Gamma}_{1}, \Omega \subset Q$-открытая область, $\Gamma_{2} \subset \partial Q$ - замкнутое множество, имеющее непустую внутренность (в топологии границы), $\Gamma_{1}=\partial Q \backslash \Gamma_{2}, b_{s}(x)=0$, $c(x, y)=0\left(x \in \Gamma_{2}, y \in \Omega\right)$. В частности, можно рассмотреть случай, когда $\Omega=Q$.

Предположим, что $b_{s}(x), c(x, y)$ неотрицательны и

$$
\sum_{s=1}^{N} b_{s}(x)+\int_{\Omega} c(x, y) d y \leqslant 1 \quad(x \in \partial Q) .
$$

Тогда (3) может быть представлено в виде (2), и условия 3)-5) вьполняются.

Действительно, пусть

$\gamma(x)=1-\sum_{s=1}^{N} b_{s}(x)-\int_{\Omega} c(x, y) d y, \quad \mu(x, G)=\sum_{s \in J(x, G)} b_{s}(x)+\int_{G \cap \Omega} c(x, y) d y$ для любого борелевского множества $G \subset \bar{Q}$, где $J(x, G)=\left\{s: \omega_{s}(x) \subset G\right\}$. Тогда нелокальное условие (3) примет вид (2), т.е.

$$
\begin{aligned}
\left(1-\sum_{s=1}^{N} b_{s}(x)-\int_{\Omega} c(x, y) d y\right) & u(x)+\sum_{s=1}^{N}\left(u(x)-u\left(\omega_{s}(x)\right)\right) b_{s}(x) \\
& +\int_{\Omega}(u(x)-u(y)) c(x, y) d y=0 \quad(x \in \partial Q) .
\end{aligned}
$$

Так как $\gamma(x)+\mu(x, \bar{Q}) \equiv 1(x \in \partial Q)$, условие 3$)$ выполняется. Пусть

$$
\Delta=\min _{1 \leqslant s \leqslant N} \rho\left(\omega_{s}\left(\Gamma_{1}\right), \Gamma_{1}\right) .
$$

Тогда выполнены условия 4), 5).

ЗАмеч АниЕ 4 . В работе [8] рассматривался оператор $A$ с нелокальным условием (2). Предполагалось, что вьполняются условия 1)-3), 5) и $\Gamma_{2}=\varnothing, \Gamma_{1}=\partial Q$. Соответственно, в примере относительно нелокальных условий (3) нак ладьвались следующие ограничения: $\Omega \subset Q, \omega_{s}(\partial Q) \subset Q$.

Автор благодарит А. Л. Скубачевского за постановку задачи и постоянное внимание к работе.

Московский государственный авиационный институт

Поступило

E-mail: skub@k803mainet.msk.su 26.04 .96

\section{СПИСОК ЦИТИРОВАННОЙ ЛИТЕРАТУРЫ}

1. Feller W. // Ann. of Math. 1952. V. 55. P. 468-519. 2. Вентцель А. Д. // Теория вероятн. и ее применения. 1959. Т. 4. С. 172-185. 3. Sato K., Ueno T. // J. Math. Kyoto Univ. 1965. V. 4. P. 529-605. 4. Taira K. Diffusion Processes and Partial Differential Equations. N. Y., London: Acad. Press, 1988. 5. Taira K. // Mem. Amer. Math. Soc. 1992. V. 99. Р. 1-65. 6. Скубачевский А. Л. // Докл. АН СССР. 1989. Т. 307. С. 287-292. 7. Скубачевский А. Л . // Докл. РАН. 1995. T. 341. C. 173-176. 8. Skubachevskii A. L. // Russian J. Math. Phys. 1995. V. 3. P. 327-360. 